This holds for all values of $a$ which do not cause the denominators to vanish. Here again it follows that any series of this last type can be summed as a bilinear form in $\sqrt{ } e$ if $a$ is an even positive integer.

The essential distinction between the $f$ and the $g$ classes can now be exhibited. Let

$$
y=\frac{x^{a+2}}{a+2}+\frac{x^{a+4}}{2(a+4)}+\frac{x^{a+6}}{2.4} \frac{(a+6)}{(a+\ldots}
$$

a series which converges for all finite values of $x$, and admits of a derivative

$$
\frac{d y}{d x}=X^{a+1}\left(1+\frac{x^{2}}{2}+\frac{x^{4}}{2 \cdot 4}+\ldots\right)=x^{a+1} \exp \left(\frac{x^{2}}{2}\right) .
$$

It follows, by integration for the range $0 \leqslant x \leqslant 1$, that

$$
\frac{1}{1+h}=1-e^{-\frac{1}{2}} \int_{0}^{1} x^{a+1} e^{x^{2} / 2} d x
$$

which can evidently be evaluated very simply by parts, if, and only if, $a$ is zero or an even positive integer. But for all other values of $a$, including the odd, or $f$, class, this integral is by no means easy to track. It has some affinity with the function

$$
P(\mu)=\int_{0}^{1} u^{\mu-1} e^{-u} d u
$$

of Saalschütz (cf. Whittaker and Watson, Modern Analysis (1915) 238 ), where $2 \mu=a+1$, but its range of integration extends from 0 to $-\frac{1}{2}$ and not from 0 to 1 .

In conclusion it may be mentioned that the method employed above to derive the relations (6) equally well applies to establish the results (4). It then exhibits the same features as would be found in the recurrence relation whereby the number of permutations of $n$ letters $a_{1}, a_{2}, \ldots, a_{n}$ may be enumerated in the case when each letter is displaced from its original position, in each permutation.

\title{
Note on Polynomial Interpolation
}

By A. C. Aitren.

The formulae of interpolation of Lagrange and Newton are easily retained in the memory if one considers a simple way in which each can be derived, for polynomials. 
xiv

First, Lagrange's formula. Suppose for simplicity that we have four values $u_{a}, u_{b}, u_{c}, u_{c l}$ of the polynomial $u_{c}$. We seek an expression for $u_{x}$, in this case a cubic polynomial, into which the data $u_{a}, u_{b}, u_{c}, u_{d}$ shall enter symmetrically. One can hardly help writing

$$
u_{x}=u_{a} \phi_{a}(x)+u_{b} \phi_{b}(x)+u_{c} \phi_{c}(x)+u_{d} \phi_{d}(x),
$$

where $\phi_{a}(x)$, etc., are cubics to be determined. The identity just written down will be true for $x=a, b, c, d$ if

$$
\begin{aligned}
\text { (i) } \quad \phi_{a}(a)=\phi_{b}(b)=\ldots \ldots=\phi_{d}(d)=1, \\
\text { (ii) } \quad \phi_{a}(b)=\phi_{a}(c)=\ldots=\phi_{d}(c)=0 .
\end{aligned}
$$

Condition (ii) shows, by the Remainder Theorem, that $\phi_{a}(x)$ has factors $x-b, x-c, x-d$, and then condition (i) shows that it must also have the constant factor $\{(a-b)(a-c)(a-d)\}^{-1}$. By symmetry we obtain at once Lagrange's formula

$$
u_{2:}=\sum_{a, b, c, d} u_{a} \frac{(x-b)(x-c)(x-d)}{(a-b)(a-c)(a-d)},
$$

and the case for a polynomial of the $n^{\text {th }}$ degree is no more complicated.

Next, Newton's formula. Here not symmetry, but order of data taken, is the guiding principle. Thus $u_{a}, u_{b}, u_{c}$ alone determine a quadratic, which takes these values for $x=a, b, c$; but if we annex a further datum $u_{d}$ the quadratic gives place to the cubic $u_{x}$. The new terms thus added to the quadratic in this way constitute a certain cubic, say $\psi_{u}(x)$. But for $x=a, b, c$ the quadratic by itself gives the right values; so that $\psi_{d}(x)$ must vanish for $x=a, b, c$, and so contains $(x-a)(x-b)(x-c)$. Thus, introducing our data one at a time, we are led to write

$u_{x}=u_{a}+(x-a) u(a, b)+(x-a)(x-b) u(a, b, c)+(x-a)(x-b)(x-c) u(a, b, c, d)$, where $u(a, b), u(a, b, c)$ and so on are to be determined. Putting $x=b$, we have from (3)

$$
u(a, b)=\frac{u_{b}-u_{a}}{b-a}
$$

the "divided difference" of $u_{a}$ and $u_{b}$; while also from (3)

$u(a, x)=u(a, b)+(x-b) u(a, b, c)+(x-b)(x-c) u(a, b, c, d)$.

Putting $x=c$ in (4), we have again

$$
u(a, b, c)=\frac{u(a, c)-u(a, b)}{c-b},
$$

z " second divided difference"; and so on. The coefficients in (3) are 
thus identified as successive divided differences, and the formula (3) itself is Newton's formula.

We can go on to prove that divided differences are symmetrical in their arguments: for example $u(a, b, c)$ is seen from our work to be the coefficient of the highest power of $x$ in the polynomial determined by $u_{a}, u_{t}, u_{c}$; and this is the same whatever the order taken. Also the $n^{\text {th }}$ divided difference derived from $n+1$ values of a polynomial of the $n^{\text {th }}$ degree is constant and independent of its arguments; for it is the coefficient of the highest power of $x$ in the polynomial, which is fixed.

\title{
A Note on Mathematical Expectation
}

\author{
By A. C. Aitken.
}

If there are $n$ coins in a bag, of any individual values but of total value $P$, and if $m$ are drawn at random, without replacement, it may be proved that the mathematical expectation, or probable value, of the draw is $m P / n$. It must be well known, though I cannot find any reference in the texts, that the same result holds if the coins are replaced after each drawing, or indeed if replacements after certain specified drawings, but not after others, are permitted.

It may therefore be worth while to prove the following result:

If there are $n$ coins, of any individual values, and of total value $P$, and if $m$ are to be drawn by any rule that does not mention individual coins (e.g. with replacements, or without replacements, or with replacements at certain agreed stages), then the probable value of the draw is $m P / n$.

Proof. The $n$ coins, regarded as possessing distinct identity (though there may be groups of the same denomination), may be denoted by $k_{1}, k_{2}, \ldots, k_{n}$.

Let the number of different possible drawings of $m$ of these, under the rules agreed upon, and order being taken into account, be $N$, and let one such order be

$$
k_{\alpha}, k_{\beta}, \ldots, k_{\mu},
$$

where $a, \beta, \ldots, \mu$ are $m$ numbers chosen from $1,2, \ldots, n$, in which 